\title{
Simultaneous Measurement of the Elemental Content and Size of Airborne Particles by Inductively Coupled Plasma Emission Spectrometry Combined with the Laser Light-Scattering Method
}

\author{
Tsutomu Nomizu, Hayato Nakashima, Yoshinori HotTa, Tomokazu TaNaka \\ and Hiroshi KAWAGUCHI \\ Department of Materials Science and Engineering, Nagoya University, Nagoya 464-01, Japan
}

\begin{abstract}
A system has been developed which can simultaneously determine the size of individual airborne particles and the content of a given element in them. An aerosol sample was passed through a laser light-scattering cell in order to measure the size of individual particles. It was then introduced into an inductively coupled plasma in order to determine the given-element content of each of the individual particles by emission spectrometry. The scattered light signal from each particle was compared with its atomic emission signal, appeared at a certain time lag. A number of pairs of both signal heights were plotted on a computer display showing the relation between the particle size and the content of the element. The measurement of a sample of monodisperse calcium acetate aerosol produced a relatively concentrated plot, while that of a polydisperse calcium acetate aerosol produced a linearly distributed plot.
\end{abstract}

Keywords Inductively coupled plasma, emission spectrometry, laser light scattering, airborne particle, monodisperse aerosol, polystyrene latex bead

Studies of the characteristics of airborne particles are essential in environmental sciences as well as in the development of air-pollution and clean-environment control technology. Airborne particle counters using laser light scattering are commercially available which can measure the particle number density as well as the size distribution in real time. The chemical composition of airborne particles, however, has usually been analyzed using time-consuming processes, in which the particles collected on a membrane or glass filter of an air sampler are dissolved before the average composition of particles can be determined using such sensitive analytical methods as inductively coupled plasma atomic emission spectrometry (ICP-AES) and atomic absorption spectrometry (AAS).

We have developed an emission spectrometric method which can determine the content of an element in individual particles by measuring the pulse height of the atomic emission signal from an ICP. ${ }^{1}$ Since the pulse height of the atomic emission signal is basically proportional to the element quantity in a particle in the micrometer to sub-micrometer range, the developed method can be used to measure the size distribution for particles having a known composition. For particles having unknown, but homogeneous compositions, the average particle diameter can be estimated by this method, which measures the decreasing rate of the particle number density due to particle sedimentation in a cylindrical sampling vessel. ${ }^{2}$ For actual aerosol samples having heterogeneous composition, however, this method can give only the

Note: This article was prepared as camera-ready copy. number density of particles containing a certain elemental quantity, not related to the particle size.

In this paper we present a combined system involving ICP-AES and the laser light-scattering method in order to simultaneously measure the content of a given element and the size of individual aerosol particles. After the scattered light of a particle in an air stream is measured, its atomic emission is measured by introducing it into the ICP. The scattered light signal for each particle corresponding to its emission signal can be plotted on a display as a twodimensional plot of the given element content against the particle size.

\section{Experimental}

\section{General description of the instrumentation}

Figure 1 shows a block diagram of the instruments used in the present work, though the section of emission spectrometry is almost the same as that in our previous work. ${ }^{2}$ A light-scattering cell was attached to a conventional concentric nebulizer for solutions, which was used to introduce aerosol samples into the ICP torch by suction from the scattering cell. The nebulizer was connected to the torch with a ball joint in order to minimize the path length between the cell and the plasma. Signals from both photomultipliers of the monochromator and scattering cell were separately amplified by a laboratory-made preamplifier and a logarithmic amplifier. Each signal was then fed to a $1 \mathrm{kHz}$ low-pass filter in order to reduce the shot noise and, further, to a laboratorymade pulse height analyzer. Each background signal was 
suppressed with each offset adjuster of the preamplifier.

Laser light-scattering cell

Figure 2 shows cross sectional views of the lightscattering cell using a semiconductor laser (Toshiba TOLD 9200 , wavelength $670 \mathrm{~nm}$, laser power $2.7 \mathrm{~mW}$ ) as a light source and a photomultiplier (Hamamatsu Photonics $\mathrm{R} 1546)$ as a detector. The semiconductor laser was preferred to a He-Ne laser for installation into a small-size scattering cell. Since the laser beam has $7 \times 34$ degrees of dispersion, a non-spherical lens head (Applied OptPlanning Inc. MINI-670FT) was attached in front of the laser (laser power decreased to $c a .1 .5 \mathrm{~mW}$ by the lens). The spot size of the laser beam was set to $0.8 \mathrm{~mm}$ at the center of the air sample stream by adjusting the lens focus. To eliminate any stray light, three thin brass plates with pinholes of $2.0,1.2$ and $1.0 \mathrm{~mm}$ diameter, respectively, were incorporated in front of the lens (in this order) at a distance of $5 \mathrm{~mm}$ each. A 0.4-mm inner diameter of the inlet and outlet tubes for an aerosol sample was selected as a compromise to obtain a higher scattering signal and less pressure resistance. A forward $60^{\circ}$ was chosen as the detection angle of the scattered light based on the work of Kanagawa et al., in which detection at smaller angles had a complicated calibration curve for the particle size, though having higher sensitivity. ${ }^{3,4}$ Although we tried to use a photodiode (Hamamatsu Photonics G1736) as a scatteredlight detector, the sensitivity was found to be insufficient.

\section{Standard polystyrene latex beads aerosol}

The laser light-scattering cell was calibrated with 0.294 , 0.506 and $1.001 \mu \mathrm{m}$ standard polystyrene latex beads (Japan Synthetic Rubber Inc. STADEX-SC-031-S, -SC051-S and -SC-101-S, respectively). After each standard particle suspension was diluted 1000-fold with water, a $2 \mathrm{ml}$ aliquot was transferred into a laboratory-made recycling nebulization chamber. The chamber had a similar structure to that described by Zhuang and Bames, and could maintain feeding of an aerosol sample into the lightscattering cell for $5 \mathrm{~min}$. To reduce the deposition of

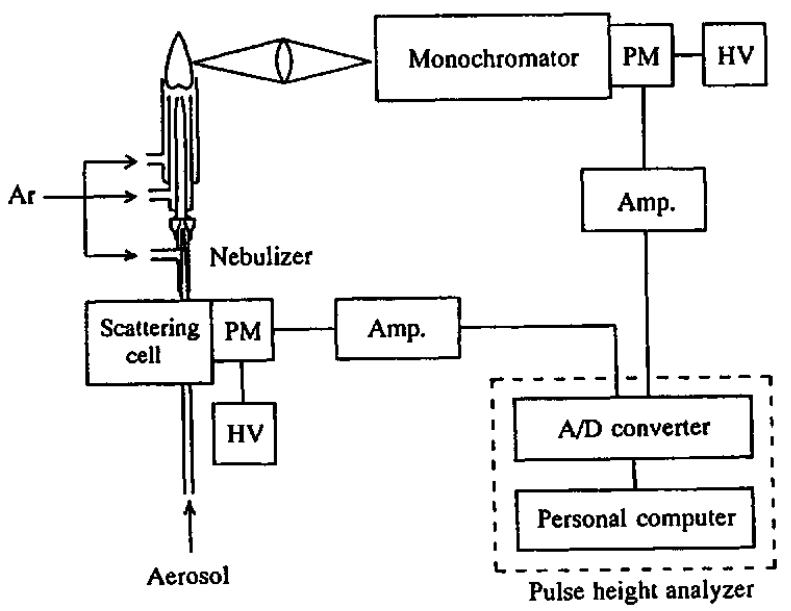

Fig. 1 Block diagram of the experimental system. charged particles on the wall of apparatus and tubes, the charge in the aerosol containing the polystyrene latex beads was effectively neutralized with bipolar ions generated by alpha-ray emission from $100 \mu \mathrm{Ci}$ of ${ }^{241} \mathrm{Am} .{ }^{6}$ The aerosol stream was further passed through a glass heating tube (20 mm o.d., $40 \mathrm{~cm}$ long) heated by Nichrome wire at $300{ }^{\circ} \mathrm{C}$ to evaporate the solvent, and through a Liebig condenser to remove any water vapor.

\section{Monodisperse aerosol of calcium acetate}

In order to calibrate the emission intensities of the $\mathrm{Ca}$ II $393.4 \mathrm{~nm}$ line for calcium-containing aerosol particles, monodisperse calcium acetate aerosols were generated by a vibrating orifice monodisperse aerosol generator (TSI Inc. Model 3450) based on the work of Berglund and Liu. ${ }^{7} \mathrm{~A}$ $10-\mu \mathrm{m}$ diameter orifice was vibrated at $240 \mathrm{kHz}$ and known concentrations of calcium acetate solution in 2propanol (50\% in water) were fed at a flow rate of $0.10 \mathrm{ml} / \mathrm{min}$. The flow rates of dispersion and dilution air for the aerosols were adjusted to 1.5 and $10 \mathrm{l} / \mathrm{min}$, respectively. The aerosol of calcium acetate was also passed through a charge neutralizer with ${ }^{241} \mathrm{Am}$, a glassheating tube and a Liebig condenser as mentioned above.

Measurement of the time lag between the light-scattered and emission signals

An aerosol generated by magnetically stirring calcium acetate powder in a beaker while blowing air was introduced into the system to measure the time lag between the scattered light and calcium emission signals derived from a single particle. Both signals from the preamplifiers
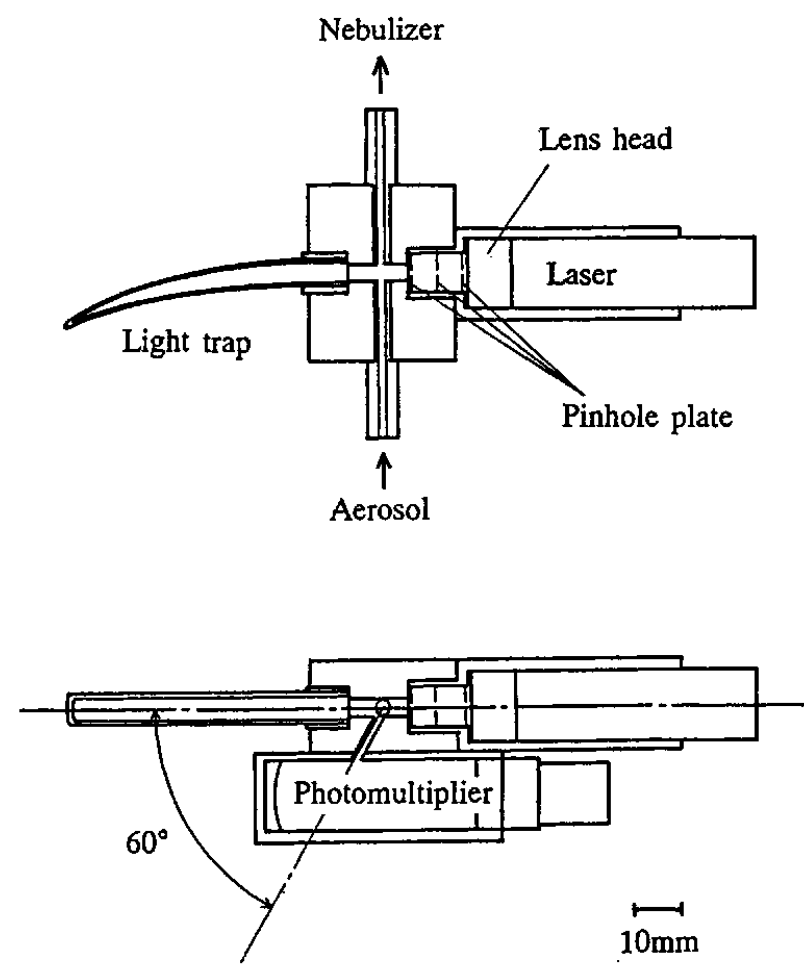

Fig. 2 Cross-sectional views of the laser light-scattering cell. 


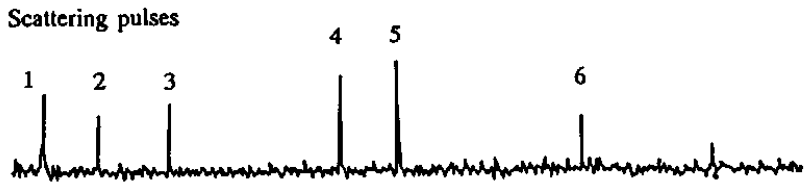

Emission pulses

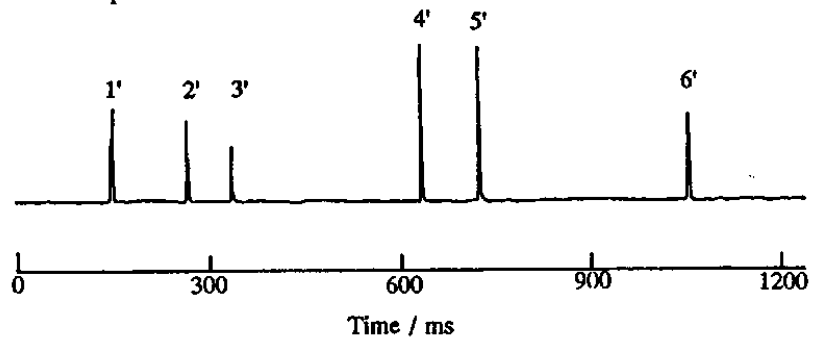

Fig. 3 Light-scattered and emission signals simultaneously stored in a two-channel digital storage oscilloscope.

were simultaneously stored in a two-channel digital storage oscilloscope (Iwatsu Electric Co. SS-5802) for a second in parallel; the stored signals were recorded on a $\mathrm{X}-\mathrm{Y}$ recorder (Watanabe Sokki WX 4402). As shown in Fig. 3, the signal patterns for scattering and emission resemble each other; scattering pulses $1,2,3, \cdots$ and 6 , reasonably correspond to emission pulses $1^{\prime}, 2^{\prime}, 3^{\prime}, \cdots$ and 6 , respectively. The time lag between each pair of pulses was measured using the distance on the recorder chart. As a result of picking up 60 pairs of such signals from recorder charts, the average time lag was calculated to be $134.4 \mathrm{~ms}$ with a standard deviation of $29.8 \mathrm{~ms}$. Since the time lag depends on the flow rate of the aerosol stream and, therefore, the nebulizer gas-flow rate. Monodisperse calcium acetate aerosol was inconvenient for use in this experiment, since it was often difficult to recognize the corresponding pairs of signals.

\section{Signal processor}

The procedure for processing the emission signals was described in our previous paper. ${ }^{1}$ Similarly, light-scattered signals from the photomultiplier followed by a preamplifier were fed to a logarithmic amplifier in order to cover a large dynamic range. Pulse heights between $30 \mathrm{mV}$ and $10 \mathrm{~V}$ were converted logarithmically into positive pulses, which were then passed through a $1 \mathrm{kHz}$ low-pass filter to reduce the shot noise. The pulse-height analysis of light-scattered signals was executed using a program written in machine language in an 8-bit computer (Fujitsu FM-8) with an 8bit $\mathrm{A} / \mathrm{D}$ converter in the same manner as in our previous work conceming emission signals.

One of two signals, light-scattered or emission, was fed alternately into the A/D converter by switching the signals with a multiplexer (DG508A). A computer program (also written in machine language) was used to search for corresponding signals of scattered light and emission from each particle, and then displaying the data graphically on a cathode ray tube.

In the first step, light-scattered signals converted to digital were successively stored in the computer memories for a preset acquisition time, close to the above-mentioned average time lag (i.e., $130 \mathrm{~ms}$ in this experiment). During this step, however, only the peak values of the pulse signals were stored, while the others were reset to zero in order to reduce the processing time at the subsequent step. Memories of $1.3 \mathrm{kbytes}$ were used in this step, i.e. the period between any two consecutive bytes of the memories corresponded to $0.1 \mathrm{~ms}$.

In the second step, the emission signals were stored in the same manner, but in another group of memories.

In the third step, pairs of the light-scattered and emission signals from a single particle were searched for by comparing the data in the two groups of memories. The number of emission pulses is usually less than that of the light-scattered pulses, since only those particles containing a given element generate an emission pulse, and some of the particles are deposited in the pathway between the light-scattering cell and the plasma torch. Therefore, the emission signals in the stored data were searched in advance from the beginning of the memories. Once an emission peak signal was found in the memories, a corresponding peak was searched in the scattering-signal memories. Since the acquisition time of both signals was set to a value close to the time lag, the corresponding signals should appear at nearly the same address of the memories from the beginning of both groups of memories. The search for the scattering signals was carried out within 100 bytes around the corresponding memory address, i.e., within a time lag of $130 \pm 10 \mathrm{~ms}$. If some scattering signals were found, the closest signal to the average time lag (134.4 ms) was chosen as a corresponding signal. Then, a pair of corresponding pulse height data were stored in the third memory group. If there was no scattering peak signal, the emission peak signal was neglected.

After checking all of the emission peak signals stored in the memories, the computer program retumed to the first step. These procedures were repeated for a preset time of 3 to $5 \mathrm{~min}$. Finally, the scattered signal heights against the corresponding emission signal heights, both stored in the third memory group, were plotted on a display of the cathode-ray tube.

\section{Results and Discussion}

\section{Calibration of the laser light-scattering cell}

Figure 4 shows pulse-height spectra of the laser lightscattered signals when standard polystyrene latex bead aerosols were introduced into the light-scattering cell. Since the relative standard deviations of the beads size have been reported to be very small $(1.53,2.01$ and $2.36 \%$ for $0.294,0.506$ and $1.001 \mu \mathrm{m}$ standard beads, respectively), the broadening of the peak width in each spectrum was considered to be mainly derived from the light-scattering cell. A small shoulder in the right-hand side of each peak 
was due to conjugated beads. The peak widths, however, were sufficiently narrow if we consider the simple structure of the scattering cell without a sheath flow, which is usually used in commercial instruments.

Each peak channel in the pulse-height spectra was plotted against the $\log$ scale of each standard bead diameter, as shown in Fig. 5. A theoretical response curve calculated by Kanagawa et al. ${ }^{4,9}$, based on the Mie scattering equation, is shown in the figure since the channel number is related to the scale of the relative scattering intensity (shown in the right of the figure) from a calibration curve of the logarithmic amplifier. The scale of the relative scattering intensity and the theoretical curve were nomalized by setting the scattering intensity of a $0.5 \mu \mathrm{m}$ standard particle to unity. As shown in Fig. 5, the plots are close to the theoretical response curve though our light source as well as optics were different from the calculation basis of Kanagawa et al.

(a)

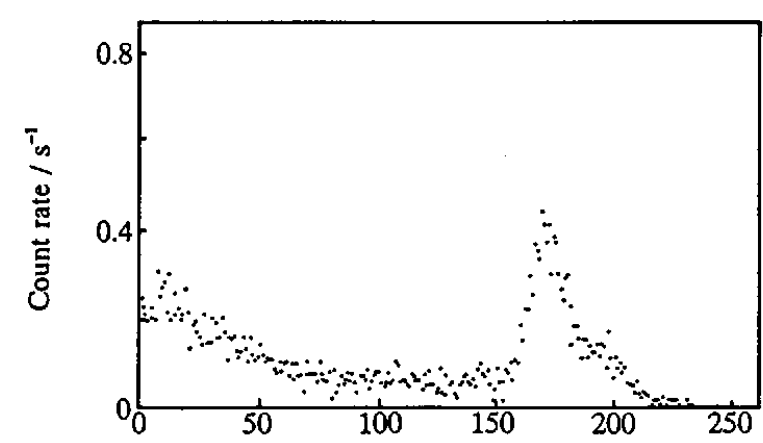

(b)

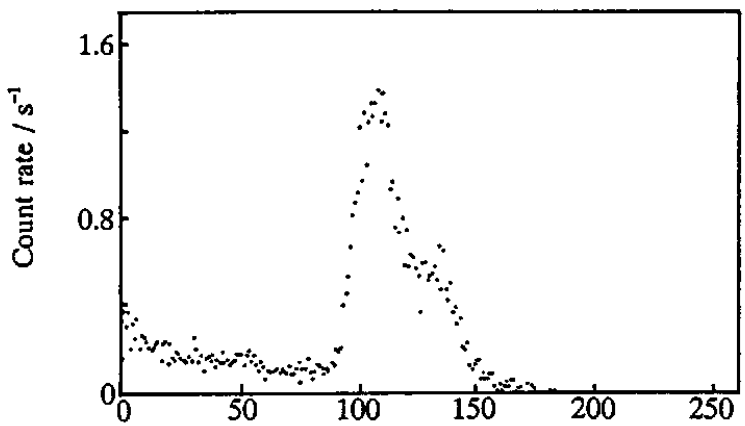

(c)

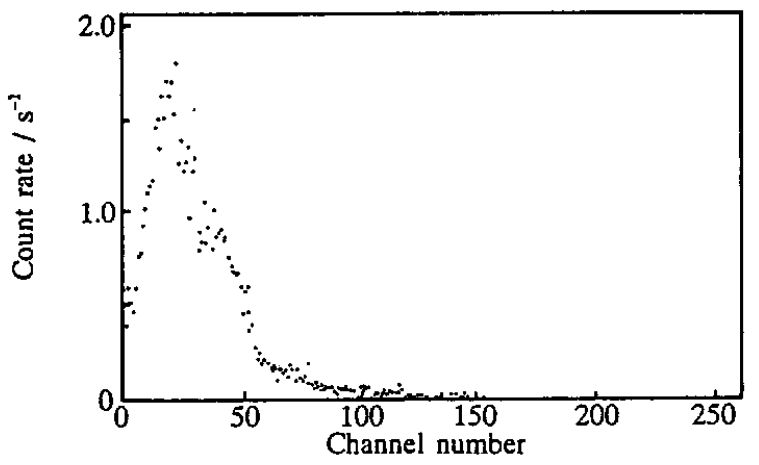

Fig. 4 Pulse-height spectra of the laser light-scattered signals obtained with standard polystyrene latex beads. (a) $1.001 \mu \mathrm{m}$, (b) $0.506 \mu \mathrm{m}$ and (c) $0.294 \mu \mathrm{m}$.
Measurement of monodisperse calcium acetate aerosol

Each calcium acetate solution of 11.4, 22.7, 45.5, and $113 \mu \mathrm{gCa} / \mathrm{ml}$ in 2-propanol (50\% in water) was fed to the vibrating orifice monodisperse aerosol generator, which generated particles of $0.76,0.96,1.22$ and $1.65 \mu \mathrm{m}$ in diameter, respectively. Figures $6(a)$ and $6(b)$ show the pulse-height spectra of the light-scattered and emission signals for a monodisperse aerosol of $1.22 \mu \mathrm{m}$ calcium acetate particles.

Plots of the emission pulse-heights against the corresponding scattered pulse-heights, i.e., the plots of the calcium content against the particle size, are shown in Fig. $6(c)$. If the diameter of aerosol particles is uniform and both measurements of the light-scattered and emission are ideal, very narrow, sharp peaks should be obtained in both pulse-height spectra, and the plots shown in Fig. 6(c) should concentrate at a point. The plots given in Fig. 6(c) have a rather wide distribution, since each pulse height spectrum had some broadening. Especially, the lower channel signals in the light scattering caused a wider horizontal distribution in the plot than the vertical one.

\section{Measurement of polydisperse calcium acetate aerosol}

A polydisperse aerosol of calcium acetate particles was also produced by the monodisperse aerosol generator by changing the orifice vibration frequency to that out of the effective range. Figure 7 shows a distribution plot for the light scattering and emission. The scale of the particle size shown in the top of the figure was obtained from the calibration curve for a light-scattered signal similar to that shown in Fig. 5, but fitted linearly for simplification. The sensitivity of the light-scattering measurement was lower in this experiment than that used for Fig. 5. The scale of

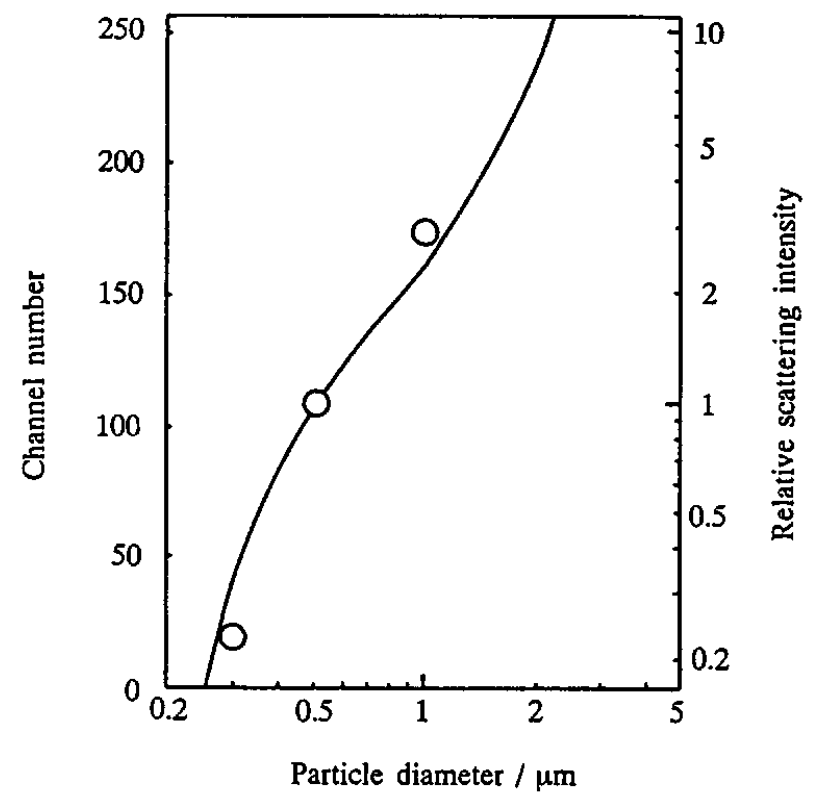

Fig. 5 Relation between the channel number and the particle diameter for a light-scattering measurement. Line is a theoretical response curve calculated by Kanagawa et al. 
(a)

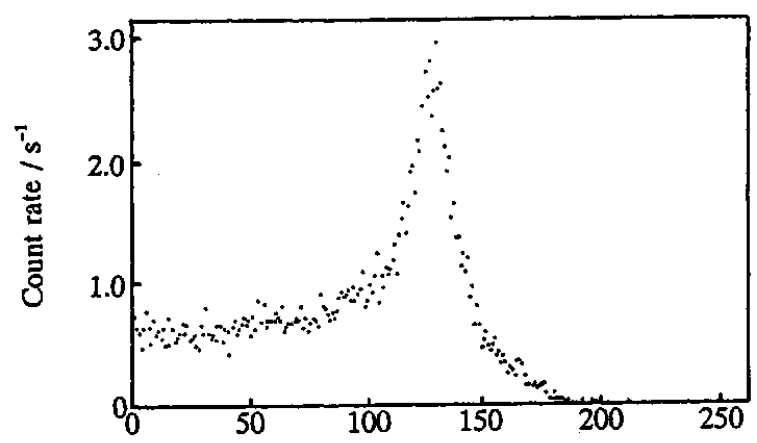

(b)

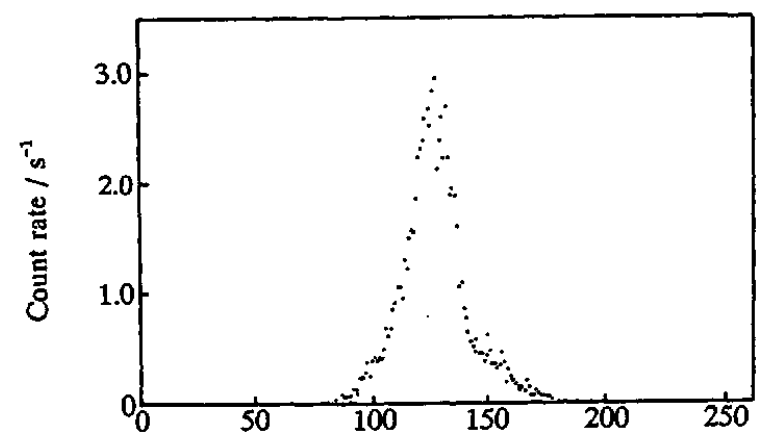

(c)

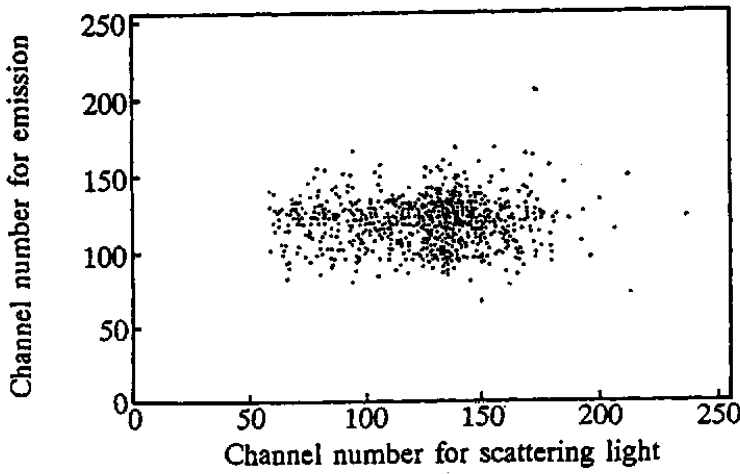

Fig. 6 (a) Pulse-height spectrum of the laser light-scattered signals, (b) pulse-height spectrum of the emission signals and (c) a plot of the corresponding pulse heights obtained with a monodisperse calcium acetate aerosol (particle diameter 1.22 $\mu \mathrm{m}$, calcium content $2.92 \mathrm{pg}$ ).

the calcium content shown in the right-hand side of the figure was obtained from the calibration curve for the emission signal of the Ca II $393.4 \mathrm{~nm}$ line.

The solid line in Fig. 7 is a calculated relation curve between the size and the calcium content for calcium acetate particles ( $\mathrm{Ca} .22 .7 \mathrm{wt} \%$ ) based on both the linear calibration curves of scattered signals and the emission signals. The broken line is the calculated relation curve for metal calcium particles (Ca $100 \mathrm{wt} \%$ ). Obviously, the plots for polydisperse calcium acetate particles are distributed along the solid line. The distribution, however, is rather broad and further modifications in the measuring system are needed before applying this method to actual aerosol samples. One of the causes of this broadening is

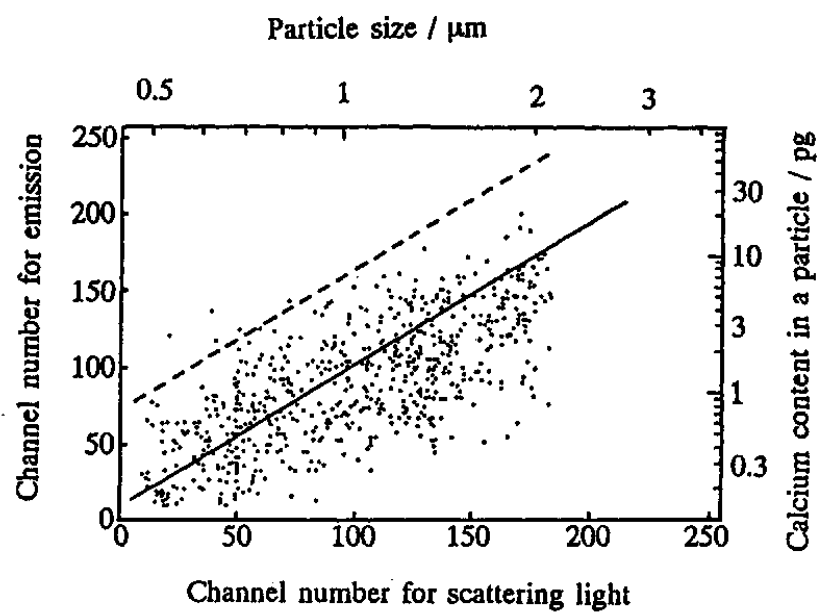

Fig. 7 Plot for corresponding pulse heights obtained with a polydisperse calcium acetate aerosol (see text for lines).

the large standard deviation of the average time lag between the light scattered and emission signals. To reduce the time lag and, therefore, to reduce the standard deviation, a more compact assembly of torch, nebulizer and light scattering cell may be required.

In the present system the size and elemental content of airborne particles in the micrometers to sub-micrometer range can be measured simultaneously in real time. A plot of the scattered signals against corresponding emission signals gave a particle size distribution related to the distribution of a given-element content. Although further modifications are necessary in the measuring system, this method will provide not only particle number density and size distribution data, but also the distribution of a givenelement content of particles for actual aerosol samples.

\section{References}

1. H. Kawaguchi, N. Fukasawa and A. Mizuike, Spectrochim. Acta, 41B, 1277 (1986).

2. H. Kawaguchi, K. Kamakura, E. Maeda and A. Mizuike, Bunseki Kagaku, 36, 431 (1987).

3. A. Kanagawa, Kagaku Kogaku, 34, 991 (1970).

4. A. Yokochi and A. Kanagawa, Kagaku Kogaku, 34, 997 (1970).

5. H. Z. Zhuang and R. Barnes, Spectrochim. Acta, 40B, 11 (1985).

6. M. Adachi, K. Okuyama, Y. Kousaka, H. Kozuru and D. Y. H. Pui, Aerosol Sci. Technol, 11, 144 (1989).

7. R. N. Berglund and B. Y. H. Liu, Environ. Sci. Technol., 7, 147 (1973).

8. W. Kaye, Anal. Chem., 45, 221A (1973).

9. A. Kanagawa, Kagaku Kogaku, 34, 521 (1970).

(Received April 9, 1992)

(Accepted June 24, 1992) 